\title{
Ultrasonic Planimals! The Bioacoustics of Fusing Cyphastrea Coral
}

\author{
Camila Rimoldi Ibanez \\ Sebring High School, 3514 Kenilworth Blvd., Sebring, Florida, 33870, USA; camilarimoldi03@gmail.com
}

ABSTRACT: Communication is vital for all organisms to grow and exchange information. The coral species Cyphastrea was tested for its hypothesized communication via ultrasonic sounds when undergoing fusion at different times of the day (morning, afternoon, nighttime). Both live and dead corals were tested with a digital oscilloscope in which the trigger level was raised above the highest peak of electrical noise to differentiate the ultrasounds emitted by the corals and the electrical noise. Because precautions towards unwanted electrical voltages were taken and the dead coral displayed no spikes in the 30 trials, it ensures that the spikes observed for the live coral were produced and emitted by the corals. Notably, $52 \%$ of all spikes occurred during the nighttime intervals in comparison to $28 \%$ during afternoon and $20 \%$ during morning intervals. Ostensibly, there may be a connection between the different growth rates of the individual corals and the number of spikes recorded for each. Still, this proposes that live coral can emit ultrasounds and more actively do so during the nighttime than any other time of the day. This could help to understand the importance of corals and make a new foundation to restore corals worldwide.

KEYWORDS: Earth and Environmental Science; Climate Science; Marine Biology; Corals; Ultrasounds.

\section{- Introduction}

Corals are one of the most prevalent saltwater lifeforms that contribute to the health of the planet. Although corals have been part of Earth's ecosystems for the past millions of years, much is still to be discovered about the coral species. There is still much to be done to help with the bleaching epidemics that are affecting corals all over the world.

Corals provide much to society by generating jobs and over billions of dollars from fish and tourism, and to the ocean by supporting $25 \%$ of sea life (home to over 1 million marine species). It is extraordinary to think corals can do all this and cover less than $1 \%$ of the ocean floor. ${ }^{1}$ The researcher investigated the ultrasonic sound emissions from fusing coral fragments. A hypothesis is being put forth to further try to understand the complex multicellular system that make up corals.

The hypothesis is that corals emit bio-acoustical ultrasonic sounds when undergoing fusion at different times of the day, predicting more activity at night than the other times of the day. There are numerous studies that led the way for this hypothesis mostly based off from a scientific breakthrough that acknowledges plants can communicate with each other in different ways including ultrasonic sounds, chemical compounds through the roots, and chemical gases through the leaves. ${ }^{2}$ Most specifically is a study that shows plants emit ultrasonic sounds with a frequency range of $20-100 \mathrm{kHz}$ and an amplitude range of $0-60 \mathrm{~dB} .{ }^{3}$ There are studies that lead researchers to consider the fact that corals may be able to make ultrasonic sounds. Similar studies that have shown that corals communicate with fish via chemical signals and that coral larvae have sensory modalities that allow them to follow the sound of the coral reef., ${ }^{45}$ Regarding the part of the hypothesis about corals emitting more ultrasounds at nighttime, research shows that corals sexually reproduce once a year precisely two hours after the moonrise, during nighttime. ${ }^{6}$ For corals to carry out an important developmental event such as sexual reproduction at night, one might expect them to be more active at nighttime as well.

With the current bleaching, pollution, climate change, and other adversities that coral reefs face, $50 \%$ of the world's coral have been lost during the last 30 years. $^{7}$ If the hypothesis presented by the researcher is demonstrated, it may help with the restoration of the fast-dying corals because if the ultrasonic sounds are harnessed and enhanced, it might facilitate developing of techniques to help the corals reproduce and grow more effectively.

\section{- Methods}

In order to test the hypothesis that the coral specie Cyphastrea have the ability to generate sound to potentially communicate with other members of its specie via ultrasonic communication at different times of the day (morning, afternoon, and nighttime), three coral fragments were tested as an experimental group. Each of the total six experimental groups were tested with a RIGOL DS1052E Oscilloscope and a Hydrophone (with Frequency Range of 20-100 khz). Each coral group was given two twelve-minute trials at each of the three specific times of the day: nighttime (7-11 pm), afternoon (2-6 pm), and morning (7-11 am), throughout the month of November. These results were compared to that of dead coral, which were tested with the same equipment and testing procedures. At the same time growth was measured and tracked, weekly, for each individual coral fragment. Although moon phases have been observed to affect corals, and specifically coral reproduction, moon phases were not taken into consideration in this investigation as the 
purpose of this research was to identify if corals can generate ultrasonic sounds.

\section{Precautions:}

There were a few basic precautions taken for variables such as the time of the day, the duration of the trials, the saltwater element levels that were directed towards both experimental groups. The saltwater element levels, and their consistent value range are, a temperature of around $25.6{ }^{\circ} \mathrm{C}$; salinity between 1.024 - $1.026 \mathrm{ppm}$; nitrate less than $30 \mathrm{ppm}$; nitrite level of 0 ; $\mathrm{pH}$ levels of 7.8- 8.1; an ammonia level of 0 ; alkalinity of 8-10 dkh; a calcium level between 380 - 450 ppm; magnesium levels between 1300-1450 ppm; light levels of 7 hours of blue light and 3 hours of white light. Due to the lack of research regarding the exact frequency or range of ultrasonic sounds corals produce, specific precautions were taken to differentiate background electrical noise from possible coral ultrasonic spikes. The precautions ensured that the researcher knew exactly what was producing the ultrasonic spikes as they were applied to both dead control coral and live coral experimental groups. The first of the precautions taken was to turn all the power of surrounding devices off, so that there were no unexpected electrical charges affecting the measurements. Secondly, after the average noise level created by electrical connections between the Oscilloscope and the Hydrophone were determined, the trigger level on the Oscilloscope was raised to double the average noise level. Thus, the Oscilloscope would display a signal only when it passed the trigger level and hence, surpass all electrical noise.

Besides setting up the RIGOL DS1052E Oscilloscope to display a signal only when it passed the trigger level, other specifications were set up on this device. First, Channel 1 of the two on the Oscilloscope was turned on so that the only signal displayed was the one received through the hydrophone. Then, setting Channel 1 Coupling to AC. This way it corresponds to the hydrophone input. Secondly, the Fast Fourier Transform (FFT) system was turned on throughout the experimentation. The FFT system is a combination of sine waves that represent the frequency and amplitude of the signal. By having this feature on throughout the trials, the Oscilloscope will give indications of the different characteristics of the ultrasonic spikes. To analyze these characteristics properly set the frequency as the subcategory of time. Lastly, the Trigger Level were set to Normal to enable the researcher to increase the Trigger Level to that of twice of the electrical noise.

\section{Positive Control Group:}

With these experiments it was clear that the hydrophone worked as expected and that it could capture the expected range of ultrasonic sounds. The Ultrasonic Cleaning Machine that was used as the source of ultrasounds for these experiments emitted ultrasounds at a frequency of around $40 \mathrm{kHz}$. The oscilloscope displayed frequencies at or very near to $40 \mathrm{kHz}$, only ranging from $34 \mathrm{kHz}$ (the lowest recorded frequency) to 49 $\mathrm{kHz}$ (the highest recorded frequency). The consistency of the rectangular wave that the oscilloscope produced based of the signal received through the hydrophone acts as the final check for everything working properly.

\section{Results and Discussion}

As can be seen in Figure 1, the average noise level does not surpass the $\sim 15 \mathrm{mV}$ trigger level, this is concluded since throughout the trial there were no ultrasonic electrical noise spikes that passed this trigger level. As a result, when testing the live and dead coral the trigger level was set to $~ 35-40$ $\mathrm{mV}$ which is more than double of the trigger level that the background noise level did not pass. This guaranteed that any ultrasonic spikes observed would be coming from the testing group and not from background noise. Therefore, since the only variable between control group and testing group was whether the corals were alive or dead, any spikes would represent ultrasonic sounds emitted by either group. Because in this investigation the dead control group corals did not have any spikes throughout the 6 hours of the 30 trials conducted, it strengthens the conclusion that the spikes observed during the measurement of the experimental groups were emitted by the live corals.

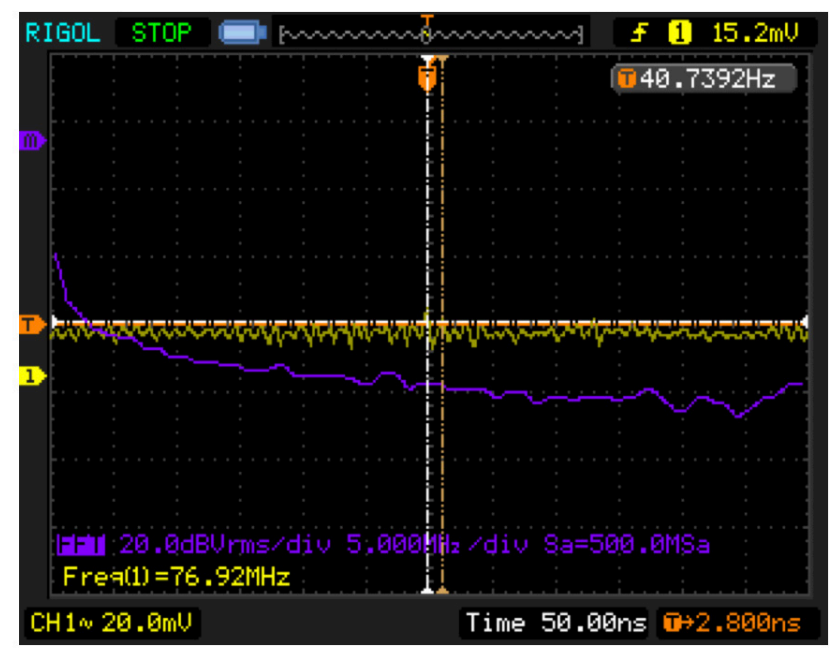

Figure 1: Control Coral \#5, Afternoon Trial 3.

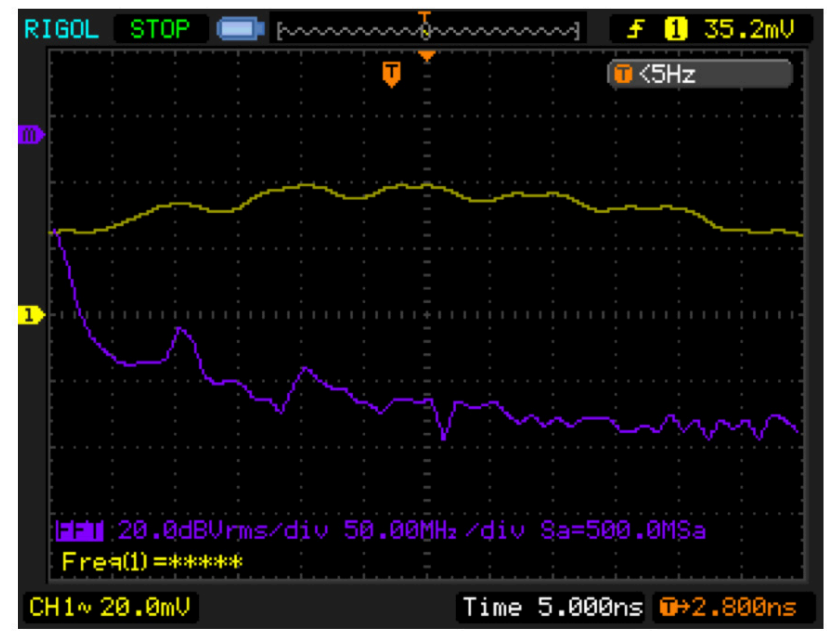

Figure 1: Control Coral \#5, Afternoon Trial 3.

\section{Ultrasonic Spikes:}

The most important aspect tested in this investigation was whether corals had the ability to emit ultrasonic waves and potentially communicate with each other. This appears to be true 
as there was a total of 25 ultrasonic spikes recorded from the Live Coral Testing Groups while no spikes were recorded for the dead corals. After recording the spikes, they were analyzed with the time of day they were measured as well as the growth rates of the individual corals (see example in Figure 2).

There was a strong connection between the different times of the day and the number of spikes. When considering all spikes, there were a total of 13 spikes during the nighttime intervals (7-11 pm), 7 spikes during the afternoon intervals (2-6 $\mathrm{pm})$, and 5 spikes during the morning intervals (7-11 am). All of these spikes had an average intensity of $47.81 \mathrm{MHz}$. On average, the live corals emitted $62 \%$ more ultrasounds during the nighttime intervals than during the morning. Likewise, the live corals emitted $46 \%$ more ultrasounds during the nighttime intervals than during the afternoon. The corals were significantly more active during the nighttime recordings than other times of the day. The afternoon recordings proved to be the time of the day, second to nighttime, in which corals emitted most ultrasounds. The live corals emitted $30 \%$ more ultrasounds during the afternoon intervals than morning. On average, 52 $\%$ of all spikes occurred during the nighttime intervals, $28 \%$ during the afternoon, and $20 \%$ during morning (Figure 3). The standard deviation for the different number of spikes at the three testing intervals is 4.16 and considering the mean of the same values is 8.33 , double the standard deviation, there is significant difference between the time of day and number of associated ultrasonic spikes.

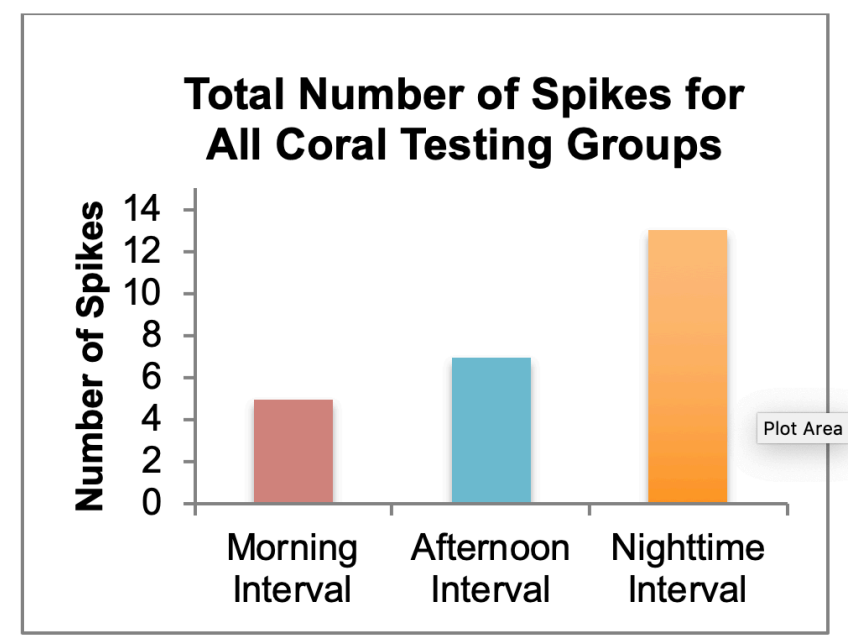

Figure 3: Total number of spikes for all coral testing groups. Nighttime spikes are more prevalent.

When independently analyzing each experimental group and their corresponding spikes, similar results are observed (Figure 4). In 2 out of 6 experimental groups, the nighttime intervals had more spikes than morning and afternoon. Although also in 2 out of the 6 experimental groups, all three intervals had the same number of spikes: 1 per interval, 3 spikes total. The afternoon interval was the interval with most spikes for 1 of the 6 testing groups, this was not seen for any of the morning intervals. Therefore, the nighttime intervals had the most spikes when independently observing the experimental groups as well as were the most prominent in overall number of spikes as $52 \%$ of all spikes occurred during the nighttime intervals.
Table 1: The ultrasonic spikes observed during the three testing daytime intervals in correspondence to the experimental group from which they were observed from.

\begin{tabular}{|c|c|c|c|c|}
\hline & Morning Interval & Afternoon Interval & Nighttime Interval & Total \\
\hline Testing Group *11 & None & 1 & 1 & 2 \\
\hline Testing Group \#2 & 1 & 4 & 1 & 6 \\
\hline Testing Group $\# 3$ & None & None & 6 & 6 \\
\hline Testing Group \#4 & 1 & 1 & 1 & 3 \\
\hline Testing Group $* 5$ & 1 & 1 & 1 & 3 \\
\hline Testing Group $\# 6$ & 2 & None & 3 & 5 \\
\hline Total & 5 & 7 & 13 & \\
\hline
\end{tabular}

Secondly, corals' spikes were compared to the growth rates. There seems to be a smaller connection between spikes and the growth rates of the corals as there was with the times of days. All corals showed signs of growth at some point in the 7-week period experimentation. However, 3 of the 18 live corals did start to shrink because they started to die. The rate of growth is seen in the line graphs, the percentages represent an average of the growth in length and height of the corals. These percentages are related to the original size of the coral. All growth rates start at $100 \%$, as this represents that the corals started with $100 \%$ of their original surface area. For example, experimental group \#2, Coral D is seen to have $12.5 \%$ growth increase by Week 7. To calculate this percentage the difference between the percentage point for Week $7(\sim 112.5 \%)$ and the initial $100 \%$ point is calculated, resulting in $12.5 \%$, and since it is positive it means the coral grew. Coral $\mathrm{M}$ in experimental group \#4 on the other hand, is seen to have lost surface area as the difference between the percentage point for Week $7(\sim 45$ $\%)$ and the initial $100 \%$-point results in a $-55 \%$ change.

The measurements did not show similar growth trends for all three corals in each testing tile. This is seen in experimental group \#2 (Figure 4). By Week 7, Coral D had grown a 12.5 $\%$, Coral $\mathrm{E}$ a $15 \%$, and Coral $\mathrm{F}$ a $34.19 \%$ - more than half of the other corals on the same tile. This relation between the different corals of the same tiles were evident in the two tiles that had dying corals. While some of the testing group corals in a tile showed significant growth rates the third coral was decreasing its size. For instance, in Testing Group \#4 (Figure 5), Coral M showed a $45.10 \%$ decrease of the original sizethis means that the coral lost about $55 \%$ of its original size. The other corals on this tile only showed growth such as Coral $\mathrm{N}$ with a $4.17 \%$ growth and Coral $\mathrm{O}$ with $15.34 \%$ growth. Both tiles with dying corals also had corals that only showed signs of growth, this reinforces how the tiles were not consistent with growth trends. Live Coral Testing Groups \#2 and \#3 both had a total of 3 spikes each and yet Testing Group \#2 had Coral M, was one of the dying corals, that lost $55 \%$ of its original size (Figure 4) and Testing Group \#3 had Coral K, the coral with most growth. The same observation was observed for experimental group \#5. This tile had two of the three dying corals of this investigation and yet had the second greatest number of spikes. Experimental group \#5 had a total of five spikes, however, it had Coral $\mathrm{P}$ which lost $48 \%$ of its original size and Coral Q which lost $67 \%$ of its original size (Figure 6). 


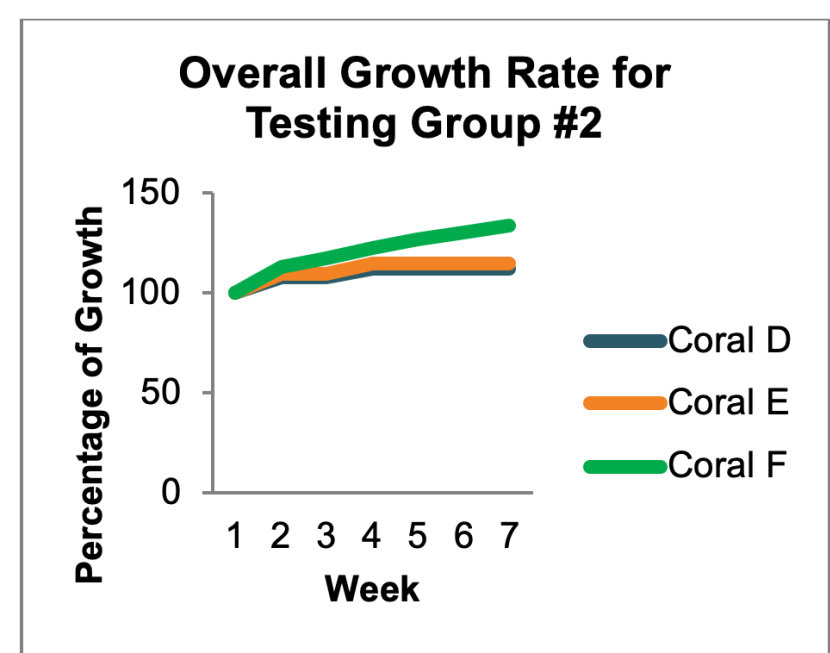

Figure 4: Percent of Coral Growth over Time for Testing Group \#2. All corals grew slightly or stayed the same.

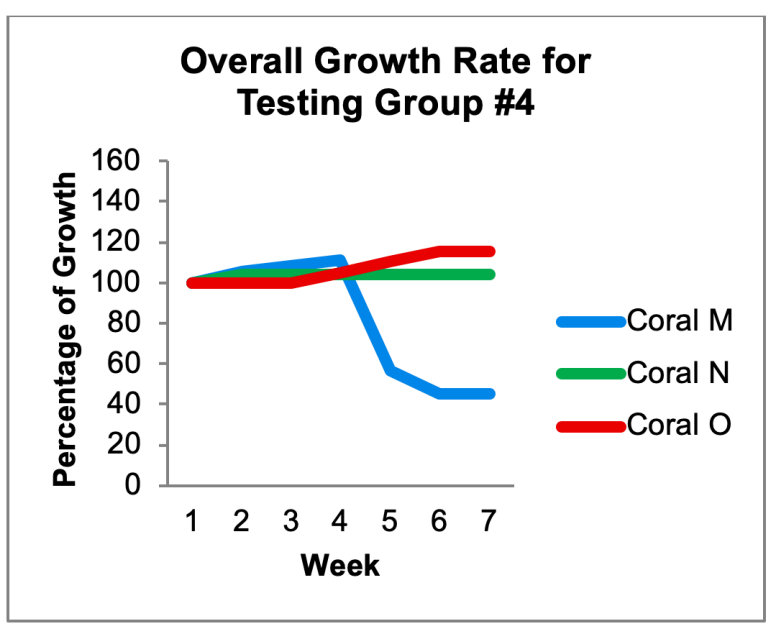

Figure 5: Percent of Coral Growth over Time for Testing Group \#4. Coral $\mathrm{M}$ decreased in size.

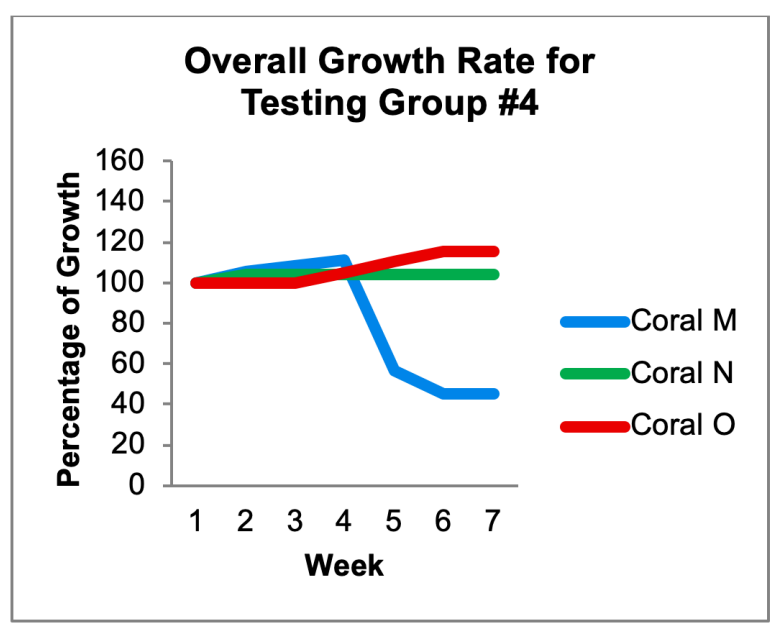

Figure 6: Percent of Coral Growth over Time for Testing Group \#5. Second Most Spikes present.

\section{Conclusion}

After conducting a total of 66 trials, the hypothesis has been partially shown to be correct. The coral fragments emitted $52 \%$ of all the ultrasonic spikes during the nighttime intervals. The first important aspect to acknowledge is that corals can emit ultrasonic sounds. There has been new research about plants communicating with ultrasounds but none for corals, and so recognizing the corals' potential to do this might give corals the ability to make sounds. Whether the sound generation is related to the coral polyp or the zooxanthellae algae that lives within the coral cannot be determined from this investigation but is a future research endeavor in better understanding coral ultrasonic sounds. Due to the fact that special precautions were taken to distinguish noise from possible ultrasonic spikes and the 5 individual dead corals tested did not display any ultrasonic spikes throughout any of the 30 trials while the live coral testing groups did display multiple ultrasonic spikes, it is concluded from this investigation that the coral specie Cyphastrea has the ability to generate ultrasonic sounds, and potentially enable some kind of communication among other corals of its species. A second aspect that is important to acknowledge is that communication is the exchange of information between living organisms, irrespective to their level of biological organization. ${ }^{8}$ Although the ultrasounds that were observed by the corals of this investigation were affected by the different times of the day, the purpose of the ultrasounds portrayed is not known.

However, with this newly discovered potential for information exchange in corals much is to be discovered, such as what the corals are communicating and how they are doing so. Something similar occurred with the micro-fusion method. Researcher Dr. Vaughan and his team in the MOTE International Center for Coral Reef Research and Restoration discovered that when corals are cut into smaller pieces their growth rates increase significantly, hence the start of the micro-fusion method which is now the foundation of numerous restoration projects. This could equally happen with the study of communication among corals and, ultimately, develop techniques to encourage communication among corals to promote health and growth of coral reefs around the world. Likewise, as the emission of ultrasounds is further studied it may lead to other discoveries.

\section{Acknowledgement}

I would like to thank Mrs. Andrea Ibanez de Rimoldi for her assistance in getting the supplies for this experimentation and for her help editing the written sections.

\section{References}

1. About Coral Reefs. https://www.icriforum.org/aboutcoral-reefs/ (accessed Nov 2019).

2. Cossins, D. Plant Talk. http://www.the-scientist.com/ features/plant-talk-38209 (accessed Nov 2019).

3. Veits, M.; Khait, I.; Obolski, U.; Zinger, E.; Boonman, A.; Goldshtein, A.; Saban, K.; Ben-Dor, U.; Estlein, P.; Kabat, A.; Peretz, D.; Ratzersdorfer, I.; Krylov, S.; Chamovitz, D.; Sapir, Y.; Yovel, Y.; Hadany, L. Flowers respond to pollinator sound within minutes by increasing nectar sugar concentration. http://www.biorxiv.org/content/10.1101/507319v1.full (accessed Oct 2019). 
4. Amid, C.; Olstedt, M.; Gunnarsson, J. S.; Le Lan, H.;

Tran Thi Minh, H.; Van den Brink, P. J.; Hellström, M.;

Tedengren, M. Additive effects of the herbicide glyphosate

and elevated temperature on the branched coral Acropora

formosa in Nha Trang, Vietnam.

http://www.ncbi.nlm.nih.gov/pmc/articles/

PMC5978828/ (accessed Sep 2019)

5. Puyana, M. www.eolss.net/Sample-Chapters/C06/ E6-52-04-04.pdf (accessed Nov 2019).

6. Dr. David Vaughan, senior scientist and head of Coral Restoration program in the Florida Keys

7. Loria, K. The world's oceans are in even worse shape than we thought. http://www.businessinsider. com/new-studies-show-oceans-losing-oxygen-rapidcoral-bleaching-2018-1 (accessed Oct 2019).

8. Gagliano, M. Green symphonies: a call for studies on acoustic communication in plants. https://doi.org/10.1093/ beheco/ars206 (accessed Sep 2019).

\section{- Authors}

Camila Rimoldi Ibanez, a senior high school student from Sebring, Florida, and admires Florida's oceanic ecosystems. Moving from Argentina, she took advantage of the opportunity to study in Florida by learning about her new home, leading to her interest in ocean life and corals. She is currently working on her high school diploma and A.A. degree, she aspires to attain a $\mathrm{PhD}$ in Marine Biology. 